\title{
INTERPOLATION THEORY FOR THE HK-FOURIER TRANSFORM
}

\author{
JUAN H. ARREDONDO AND ALFREDO REYES
}

\begin{abstract}
We use the Henstock-Kurzweil integral and interpolation theory to extend the Fourier cosine transform operator, broadening some classical properties such as the Riemann-Lebesgue lemma. Furthermore, we show that a qualitative difference between the cosine and sine transform is preserved on differentiable functions.
\end{abstract}

\section{INTRODUCTION}

We shall deal with real Banach spaces denoted by $X$ and with their complexification given by $X+i X$. Also, given two Banach spaces $X$ and $Y$, we denote by $\mathcal{L}(X, Y)$ the Banach space of all bounded linear operators $T: X \rightarrow Y$ with the operator norm given by $\|T\|_{\mathcal{L}(X, Y)}=\sup \left\{\|T(x)\|_{Y}:\|x\|_{X} \leq 1\right\}$. For any $T \in \mathcal{L}(X, Y)$ we define

$$
\tilde{T}(x+i y):=T(x)+i T(y) \quad(x, y \in X) .
$$

It follows that $\|T\|_{\mathcal{L}(X, Y)}=\|\tilde{T}\|_{\mathcal{L}(X+i X, Y+i Y)}$. This procedure has been used by several authors [24, 2, 17].

We recall that for any $p \in[1, \infty)$ and $X \subset \mathbb{R}$, the symbol $\mathcal{L}^{1}(X)$ denotes the space of all Lebesgue measurable functions $f: X \rightarrow \mathbb{R}$ with

$$
\|f\|_{\mathcal{L}^{p}}:=\left(\int_{X}|f(x)|^{p} d x\right)^{1 / p}<\infty .
$$

Moreover, we denote by $\mathcal{W}_{p}=\{f: \mathbb{R} \rightarrow \mathbb{R} \mid f(x)=0$ a.e. $\} \equiv$ the subspace of $\mathcal{L}^{p}(X)$ on which $\|\cdot\|_{\mathcal{L}^{p}}$ vanishes. It is known that $\|\cdot\|_{\mathcal{L}^{p}}$ is a seminorm for all $p \in[1, \infty)$ and induces a norm on the quotient space $\mathcal{L}^{p}(X) / \mathcal{W}_{p}$, under which it is complete. We will denote this space with respect to its norm by $L^{p}(X)$, [27. Similarly, for $p \in[1, \infty)$ we define $\mathcal{L}^{p}(X, \mathbb{C})$ and $L^{p}(X, \mathbb{C})$ by considering functions $f: X \rightarrow \mathbb{C}$.

2020 Mathematics Subject Classification. 42A38, 46B70, 26A39, 26 A42.

Key words and phrases. Integral transforms, Fourier Analysis, Interpolation theory, Generalized integration.

The authors acknowledge the support of Mexico's Consejo Nacional de Ciencia y Tecnología (CONACYT). 
For $p=\infty$ and $f: X \rightarrow \mathbb{R}$, we define $\|f\|_{\infty}$ as the essential supremum of $|f|$, and $\mathcal{L}^{\infty}(X)$ is the vector space of all Lebesgue measurable functions $f$ for which $\|f\|_{\infty}<\infty$. Similarly, we define $L^{\infty}(X), \mathcal{L}^{\infty}(X, \mathbb{C})$ and $L^{\infty}(X, \mathbb{C})$. If $A \subsetneq X$ is a Lebesgue measurable set and $m$ denotes the Lebesgue measure, then given a Lebesgue measurable function $f$ defined on $A$ such that $m(X \backslash A)=0$ we will denote by the same symbol $f$ the trivial extension of $f$ to a (measurable) function on $X$. Furthermore, for a function $f \in \mathcal{L}^{p}(X)$ or $f \in \mathcal{L}^{p}(X, \mathbb{C})$, we will call by the same symbol $f$ the (unique) element that defines this function in $L^{p}(X)$ or in $L^{p}(X, \mathbb{C})$, respectively. Also, the characteristic function of a set $E$ is given by $\chi_{E}(x)=1$ if $x \in E$ and zero otherwise.

If $f$ belongs to $L^{1}(\mathbb{R}) \cap L^{p}(\mathbb{R})$, the Fourier transform is defined for every real number $s$ as

$$
\begin{aligned}
\mathcal{F}_{p}(f)(s) & :=\int_{\mathbb{R}} e^{-i s x} f(x) d x \\
& =\int_{\mathbb{R}} \cos (s x) f(x) d x-i \int_{\mathbb{R}} \sin (s x) f(x) d x \\
& =\mathcal{F}_{p}^{c}(f)(s)-i \mathcal{F}_{p}^{s}(f)(s),
\end{aligned}
$$

where the integral is taken in the Lebesgue sense. $\mathcal{F}_{p}^{c}$ and $\mathcal{F}_{p}^{s}$ are called Fourier cosine and Fourier sine transforms, respectively. Furthermore, by interpolation theory, the operator $\mathcal{F}_{p}(f)$ is extended to $L^{p}(\mathbb{R})$ for $p \in[1,2]$ as a bounded operator

$$
\mathcal{F}_{p}: L^{p}(\mathbb{R}) \longrightarrow L^{q}(\mathbb{R})
$$

with

$$
\left\|\mathcal{F}_{p}(f)\right\|_{p} \leq \gamma_{p}\|f\|_{q}
$$

where $1 / p+1 / q=1$ and

$$
\gamma_{p}= \begin{cases}1 & \text { if } p=1 \\ (2 \pi)^{\frac{1}{q}}\left(\frac{p-1}{p}\right)^{\frac{p-1}{2 p}} p^{\frac{1}{2 p}} & \text { if } 1<p \leq 2 .\end{cases}
$$

The value of $\gamma_{p}$ is given by the Hausdorff-Young inequality [25], the sharp HausdorffYoung inequality [5, 29], [15, Theorem 5.7] and [3].

For any unbounded subset $X \subset \mathbb{R}$, the space $C_{\infty}(X)$ denotes the complex valued continuous functions on $X$ vanishing at infinity [25]. We denote the space of bounded variation functions by $B V(\mathbb{R})$ and by $B V_{0}(\mathbb{R})$ the subspace of functions vanishing at infinity, $\left[12,4,31\right.$. Also $B V_{0}(\mathbb{R}, \mathbb{C})$ is the corresponding complexification of $B V_{0}(\mathbb{R})$.

In [30] the Henstock-Kurzweil integral was employed to study the Fourier transform. In [20, 22] it was proved that (1.1) makes sense as a Henstock-Kurzweil integral on $B V_{0}(\mathbb{R})$. In fact, we have the following statement in 23$]$. 
Definition 1.1. The HK-Fourier transform exists for every $s \neq 0$, and is defined by

$$
\begin{aligned}
& \mathcal{F}_{H K}: \mathcal{L}^{1}(\mathbb{R})+B V_{0}(\mathbb{R}) \rightarrow C_{\infty}(\mathbb{R} \backslash\{0\}), \\
& \mathcal{F}_{H K}(f)(s):=\int_{-\infty}^{\infty} e^{-i s x} f(x) d x,
\end{aligned}
$$

where the integral is in the Henstock-Kurzweil sense. Analogously, we define the HK-Fourier cosine transform $\mathcal{F}_{H K}^{c}$ and the HK-sine Fourier transform $\mathcal{F}_{H K}^{s}$ as in (1.1.

We say "HK-Fourier transform" in order to emphasize the use of the HenstockKurzweil integral [30. Moreover, $\mathcal{F}_{H K}(f)(s)$ is pointwise defined and is continuous except at zero; see example $3(\mathrm{~d})$ in [30. Note that $\mathcal{F}_{H K}$ is well defined because the Henstock-Kurzweil integral contains the Lebesgue integral, [14, 19]. $\mathcal{F}_{1}$ can be seen as an extension of the HK-Fourier transform restricted to $B V_{0}(\mathbb{R})$,

$$
\mathcal{F}_{H K}: B V_{0}(\mathbb{R}) \rightarrow C_{\infty}(\mathbb{R} \backslash\{0\}) .
$$

Moreover, $\mathcal{F}_{p}$ is an extension of $\mathcal{F}_{1}$, so that $\mathcal{F}_{p}$ is an extension of $\mathcal{F}_{H K}$.

The relation between $\mathcal{F}_{p}$ and $\mathcal{F}_{H K}$ was first studied in [23], while the operator $\mathcal{F}_{H K}^{c}$ was studied in [3]. This work builds on these references.

\section{Henstock-KurZweil Fourier transform}

The space of Henstock-Kurzweil integrable functions defined on an interval $I$ is denoted by $\mathcal{H} \mathcal{K}(I)$. This space is a seminormed space with the Alexiewicz seminorm, defined as

$$
\|f\|_{\mathcal{H} \mathcal{K}}=\sup \left\{\left|\int_{c}^{d} f(x) d x\right|:[c, d] \subset I\right\} .
$$

The quotient space $\mathcal{H} \mathcal{K} / \mathcal{W}(I)$ will be denoted by $H K(I)$, where $\mathcal{W}(I)$ is the subspace of $H K(I)$ for which the Alexiewicz seminorm vanishes [7]. The completion will be denoted by $\widehat{H K}(I)$ and its complexification will be written as $\widehat{H K}(\mathbb{R}, \mathbb{C})$.

We study the HK-Fourier cosine transform defined by

$$
\mathcal{F}_{H K}^{c}(f)(s)=\int_{-\infty}^{\infty} \cos (s x) f(x) d x \quad(s \neq 0) .
$$

Notice that for $s=0$ and $f \in B V_{0}(\mathbb{R}), \mathcal{F}_{H K}^{c}(f)(0)$ might not be defined. Also, we have that

$$
\mathcal{F}_{1}^{c}(f)(s)=\mathcal{F}_{H K}^{c}(f)(s)
$$

for all $f \in \mathcal{L}^{1}(\mathbb{R}) \cap B V_{0}(\mathbb{R})$ and $s \in \mathbb{R}$. However, a partial result about the question of continuity at $s=0$ was proved in [3, Theorem 1]. In fact, $\mathcal{F}_{H K}^{c}$ is bounded while $\mathcal{F}_{H K}^{s}$ is not. Actually, Theorem 1 and Proposition 3 in 3 . imply the following statement. 


\section{Theorem 2.1.}

(i) The HK-Fourier cosine transform is a bounded linear operator from $B V_{0}(\mathbb{R})$ into $H K(\mathbb{R})$.

(ii) The Fourier transform is a densely defined closed operator from $L^{2}(\mathbb{R})$ into $H K(\mathbb{R})$.

We shall show that differences and similitudes between the Fourier cosine and Fourier sine transforms also hold on the classical Sobolev space $W^{1,1}(\mathbb{R})$. It is expected that these transforms are bounded operators with the same domain and codomain for functions with enough smoothness, for example as in the Schwartz space [25]. See also [16].

\section{INTERPOLATION THEORY}

We consider a couple $(X, Y)$ of complex Banach spaces such that $X$ and $Y$ are continuously embedded in a Hausdorff topological vector space $V$, i.e., $X \subset V$ and $Y \subset V$ with continuous inclusion. This couple is called a complex interpolation couple. In this case the intersection $X \cap Y$ is a linear subspace of $V$, and it is a Banach space under the norm

$$
\|v\|_{X \cap Y}=\max \left\{\|v\|_{X},\|v\|_{Y}\right\}
$$

The sum $X+Y=\{x+y: x \in X, y \in Y\}$ is a linear subspace of $V$ and it is endowed with the norm

$$
\|v\|_{X+Y}=\inf \left\{\|x\|_{X}+\|y\|_{Y}: x \in X, y \in Y, x+y=v\right\} .
$$

Remark 3.1. It follows from [18 that the space $X+Y$ is isometric to the quotient space $(X \times Y) / D$, where $D=\{(d,-d) \in X \times Y: d \in X \cap Y\}$. Since $V$ is a Hausdorff space, $D$ is closed, so $X+Y$ is a Banach space. Moreover, $X$ and $Y$ are continuously embedded in $X+Y$.

Throughout this section we shall consider $\mathbb{S}=\{z \in \mathbb{C}: 0 \leq \operatorname{Re}(z) \leq 1\}$ and we shall use the complex space $X+Y$ and the space $\mathbb{F}(X, Y)$ of functions $f: \mathbb{S} \rightarrow X+Y$ holomorphic on the interior of the strip $\mathbb{S}$ and continuous up to its boundary, such that the maps $t \mapsto f(i t)$ and $t \mapsto f(1+i t)$ are continuous from the real line into $X$ and $Y$, respectively. Therefore, $\mathbb{F}(X, Y)$ is a Banach space with the norm given by

$$
\|f\|_{\mathbb{F}}:=\max \left\{\sup _{t \in \mathbb{R}}\|f(i t)\|_{X}, \sup _{t \in \mathbb{R}}\|f(1+i t)\|_{Y}\right\}<\infty .
$$

These facts can be consulted in [18, Ch. 2], [6, Ch. 4], [9, Ex. 2.6.6], 28, Ch. 2], [13, Ch. 4] and [10, 1-4].

Definition 3.2. For every $\theta \in(0,1)$, the space $[X, Y]_{\theta}$ consists of all $a \in X+Y$ such that $a=f(\theta)$ for some $f \in \mathbb{F}(X, Y)$ and the norm on $[X, Y]_{\theta}$ is

$$
\|a\|_{[\theta]}=\inf \left\{\|f\|_{\mathbb{F}}: f(\theta)=a, f \in \mathbb{F}(X, Y)\right\} .
$$

Remark 3.3. The space $X \cap Y$ is dense in $[X, Y]_{\theta}$ and $[X, Y]_{\theta}$ is isomorphic to the quotient space $\mathbb{F}(X, Y) / \mathfrak{N}_{\theta}$, where $\mathfrak{N}_{\theta}$ is the subset of $\mathbb{F}(X, Y)$ consisting of the functions vanishing at $z=\theta$. Moreover, $\mathfrak{N}_{\theta}$ is closed (see [6, 18]). 
Theorem 3.4. The space $[X, Y]_{\theta}$ is a Banach space and an intermediate space with respect to $(X, Y)$, i.e.,

$$
X \cap Y \subset[X, Y]_{\theta} \subset X+Y
$$

with continuous inclusion.

Remark 3.5. It follows from [18, Corollary 2.8, Proposition 2.10] that for each $\theta \in(0,1)$

$$
(X, Y)_{\theta, 1} \subset[X, Y]_{\theta} \subset(X, Y)_{\theta, \infty},
$$

where the spaces $(X, Y)_{\theta, p}$ are defined by the real method of interpolation. See also [6, Theorem 4.7.1].

Theorem 3.6. Let $\left(X_{1}, Y_{1}\right),\left(X_{2}, Y_{2}\right)$ be complex interpolation couples. If $T$ belongs to $\mathcal{L}\left(X_{1}, X_{2}\right) \cap \mathcal{L}\left(Y_{1}, Y_{2}\right)$, then the restriction of $T$ to $\left[X_{1}, Y_{1}\right]_{\theta}$ belongs to $\mathcal{L}\left(\left[X_{1}, Y_{1}\right]_{\theta},\left[X_{2}, Y_{2}\right]_{\theta}\right)$ for every $\theta \in(0,1)$. Moreover,

$$
\|T\|_{\mathcal{L}\left(\left[X_{1}, Y_{1}\right]_{\theta},\left[X_{2}, Y_{2}\right]_{\theta}\right)} \leq\|T\|_{\mathcal{L}\left(X_{1}, X_{2}\right)}^{1-\theta}\|T\|_{\mathcal{L}\left(Y_{1}, Y_{2}\right)}^{\theta} .
$$

In order to construct the interpolation space of $L^{1}(\mathbb{R})$ and $B V_{0}(\mathbb{R})$ we consider the space $\mathcal{L}^{1}(\mathbb{R}) \cap B V_{0}(\mathbb{R})$ with given norm $\|\cdot\|_{\mathcal{L}^{1}(\mathbb{R}) \cap B V_{0}(\mathbb{R})}:=\max \left\{\|\cdot\|_{\mathcal{L}^{1}},\|\cdot\|_{B V}\right\}$.

Lemma 3.7. $\mathcal{L}^{1}(\mathbb{R}) \cap B V_{0}(\mathbb{R})$ is a Banach space with the given norm.

Proof. Since $B V_{0}(\mathbb{R})$ is a Banach space, then given a Cauchy sequence $\left(f_{n}\right)_{n \geq 1}$ on $\mathcal{L}^{1}(\mathbb{R}) \cap B V_{0}(\mathbb{R})$ there is $f \in B V_{0}(\mathbb{R})$ such that

$$
\left\|f_{n}-f\right\|_{B V} \rightarrow 0 \quad(n \rightarrow \infty) .
$$

This yields uniform convergence of the sequence to $f$. Similarly, there exists $[\tilde{f}] \in$ $L^{1}(\mathbb{R})$ such that

$$
\left\|f_{n}-\tilde{f}\right\|_{L^{1}} \rightarrow 0 \quad(n \rightarrow \infty)
$$

It follows that there exists a subsequence $\left(f_{n_{k}}\right)_{k \geq 1}$ of $\left(f_{n}\right)_{n \geq 1}$ converging pointwise a.e. to $f$; see [27, 8]. From the fact that $\left(f_{n}\right)_{n \geq 1}$ converges uniformly to $f$, we get that $f(x)=\tilde{f}(x)$ a.e., yielding $f \in \mathcal{L}^{1}(\mathbb{R})$ and

$$
\lim _{n \rightarrow \infty} \int_{\mathbb{R}}\left|f_{n}(x)-f(x)\right| d x=0 .
$$

On the product space $\mathcal{L}^{1}(\mathbb{R}) \times B V_{0}(\mathbb{R})$ with given norm $\|(f, g)\|_{\mathcal{L}^{1} \times B V_{0}}:=$ $\|f\|_{\mathcal{L}^{1}}+\|g\|_{B V}$, we consider the quotient space $\left(\mathcal{L}^{1}(\mathbb{R}) \times B V_{0}(\mathbb{R})\right) / D$ where $D:=$ $\left\{(f,-f) \in \mathcal{L}^{1}(\mathbb{R}) \times B V_{0}(\mathbb{R}): f \in \mathcal{L}^{1}(\mathbb{R}) \cap B V_{0}(\mathbb{R})\right\}$. So, we set

$$
\mathcal{L}^{1}(\mathbb{R})+B V_{0}(\mathbb{R}):=\left(\mathcal{L}^{1}(\mathbb{R}) \times B V_{0}(\mathbb{R})\right) / D
$$

Therefore, if $a \in \mathcal{L}^{1}(\mathbb{R})+B V_{0}(\mathbb{R})$, then it is an equivalence class given by $a=$ $(f, g)+D$. Nevertheless, we shall write $a=f+g$ to simplify notation. Also, we define

$$
\|a\|_{\mathcal{L}^{1}+B V_{0}}:=\inf _{(h,-h) \in D}\|f-h\|_{\mathcal{L}^{1}}+\|g+h\|_{B V} .
$$

This is a norm, by standard arguments. Then we consider the completion of the space $\mathcal{L}^{1}(\mathbb{R})+B V_{0}(\mathbb{R})$, denoted by $\mathcal{L}^{1}(\mathbb{R}) \widehat{+B} V_{0}(\mathbb{R})$. In addition, on the product 
space $L^{1}(\mathbb{R}) \times B V_{0}(\mathbb{R})$ with the usual norm $\|([f], g)\|_{L^{1} \times B V_{0}}=\|[f]\|_{L^{1}}+\|g\|_{B V}$, we make

$$
D^{\prime}=\left\{([f],-f) \in L^{1}(\mathbb{R}) \times B V_{0}(\mathbb{R}): f \in \mathcal{L}^{1}(\mathbb{R}) \cap B V_{0}(\mathbb{R})\right\} .
$$

Due to $\mathcal{L}^{1}(\mathbb{R}) \cap B V_{0}(\mathbb{R})$ being complete, $D^{\prime}$ is closed in $L^{1}(\mathbb{R}) \times B V_{0}(\mathbb{R})$. We define

$$
L^{1}(\mathbb{R})+B V_{0}(\mathbb{R})=\left(L^{1}(\mathbb{R}) \times B V_{0}(\mathbb{R})\right) / D^{\prime} .
$$

Thus the sum space $L^{1}(\mathbb{R})+B V_{0}(\mathbb{R})$ is a Banach space with the quotient norm [26]. Its elements are equivalence classes of the form $\bar{a}=[f+g]=([f], g)+D^{\prime}$; however, we will just write $\bar{a}=f+g$. We have the following characterization.

Proposition 3.8. The space $L^{1}(\mathbb{R})+B V_{0}(\mathbb{R})$ is isometric to $\mathcal{L}^{1}(\mathbb{R}) \widehat{+B} V_{0}(\mathbb{R})$.

Proof. $f \in \mathcal{L}^{1}(\mathbb{R})$ yields $[f] \in L^{1}(\mathbb{R})$ with $\|[f]\|_{L^{1}}=\|f\|_{\mathcal{L}^{1}}$. Conversely, if $[f]$ belongs to $L^{1}(\mathbb{R})$ then there is $\tilde{f} \in \mathcal{L}^{1}(\mathbb{R})$ such that $f=\tilde{f}$ a.e. Then, for each $a=(f, g)+D \in \mathcal{L}^{1}(\mathbb{R})+B V_{0}(\mathbb{R})$, we define $\bar{a}=([f], g)+D^{\prime}$ in $L^{1}(\mathbb{R})+B V_{0}(\mathbb{R})$. We get

$$
\begin{aligned}
\|(f, g)+D\|_{\mathcal{L}^{1}+B V_{0}} & =\inf _{(h,-h) \in D}\|f-h\|_{\mathcal{L}^{1}}+\|g+h\|_{B V} \\
& =\inf _{([h],-h) \in D^{\prime}}\|[f-h]\|_{L^{1}}+\|g+h\|_{B V} \\
& =\left\|([f], g)+D^{\prime}\right\|_{L^{1}+B V_{0}} .
\end{aligned}
$$

Therefore, the map $a \mapsto \bar{a}$ from $\mathcal{L}^{1}(\mathbb{R})+B V_{0}(\mathbb{R})$ into $L^{1}(\mathbb{R})+B V_{0}(\mathbb{R})$ has dense range, due to $\mathcal{L}^{1}(\mathbb{R})$ being dense in $L^{1}(\mathbb{R})$. The map extends to an isometry from the completion $\mathcal{L}^{1}(\mathbb{R}) \widehat{+B} V_{0}(\mathbb{R})$ onto $L^{1}(\mathbb{R})+B V_{0}(\mathbb{R})$, implying the Proposition.

Therefore, we have characterized the real space $L^{1}(\mathbb{R})+B V_{0}(\mathbb{R})$. The complexification space of this space is given by

$$
L^{1}(\mathbb{R}, \mathbb{C})+B V_{0}(\mathbb{R}, \mathbb{C}):=\left(L^{1}(\mathbb{R})+B V_{0}(\mathbb{R})\right)+i\left(L^{1}(\mathbb{R})+B V_{0}(\mathbb{R})\right) .
$$

Similarly, we define the real space $L^{\infty}(\mathbb{R})+\widehat{H K}(\mathbb{R})$ and its complexification $L^{\infty}(\mathbb{R}, \mathbb{C})+\widehat{H K}(\mathbb{R}, \mathbb{C})$. We will consider complex spaces and omit the symbol $(\mathbb{R}, \mathbb{C})$ to simplify notation. Furthermore, for the complex interpolation couples

$$
\left(L^{1}, B V_{0}\right) \quad \text { and } \quad\left(L^{\infty}, \widehat{H K}\right)
$$

we say that $T$ is a bounded linear operator from $\left(L^{1}, B V_{0}\right)$ to $\left(L^{\infty}, \widehat{H K}\right)$ if and only if $T \in \mathcal{L}\left(L^{1}+B V_{0}, L^{\infty}+\widehat{H K}\right)$ such that $T \in \mathcal{L}\left(L^{1}, L^{\infty}\right)$ and $T \in \mathcal{L}\left(B V_{0}, \widehat{H K}\right)$.

We say that the complex spaces $\mathfrak{A}$ and $\mathfrak{B}$ are intermediate spaces between the couples in 3.1 if and only if

$$
\mathcal{L}^{1} \cap B V_{0} \subset \mathfrak{A} \subset L^{1}+B V_{0} \quad \text { and } \quad L^{\infty} \cap \widehat{H K} \subset \mathfrak{B} \subset L^{\infty}+\widehat{H K} .
$$

$\mathfrak{A}$ and $\mathfrak{B}$ are called interpolation spaces with respect to the couples in 3.1 if and only if $\mathfrak{A}$ and $\mathfrak{B}$ are intermediate spaces with the following property: $T \in$ $\mathcal{L}\left(L^{1}+B V_{0}, L^{\infty}+\widehat{H K}\right)$ implies that the restriction of $T$ to $\mathfrak{A}$ belongs to $\mathcal{L}(\mathfrak{A}, \mathfrak{B})$.

From Theorem 3.4 we have the interpolation spaces $\left[L^{1}, B V_{0}\right]_{\theta}$ and $\left[L^{\infty}, \widehat{H K}\right]_{\theta}$ with respect to $\left(L^{1}, B V_{0}\right)$ and $\left(L^{\infty}, \widehat{H K}\right)$ for each $\theta \in(0,1)$. We deal with the 
operators $\mathcal{F}_{1}^{c}$ and $\mathcal{F}_{H K}^{c}$ given in 1.1 and Definition 1.1 and their extensions to the complexification of the spaces. We use the same symbols for the extended operators. Then we define the operator

$$
\begin{aligned}
& \mathfrak{F}_{1}^{c}: L^{1}(\mathbb{R}, \mathbb{C})+B V_{0}(\mathbb{R}, \mathbb{C}) \longrightarrow L^{\infty}(\mathbb{R}, \mathbb{C})+\widehat{H K}(\mathbb{R}, \mathbb{C}) \\
& \mathfrak{F}_{1}^{c}(f+g)(s):=\mathcal{F}_{1}^{c}(f)(s)+\mathcal{F}_{H K}^{c}(g)(s) .
\end{aligned}
$$

Formula 3.2 is well defined on $\mathcal{L}^{1}(\mathbb{R})+B V_{0}(\mathbb{R})$. By interpolation theory, $\mathfrak{F}_{1}^{c}$ is extended to $L^{1}(\mathbb{R})+B V_{0}(\mathbb{R})$ for each $s \neq 0$. Thus, from Theorem 2.1 and Theorem 3.6 we conclude that

$$
\mathfrak{F}_{1}^{c} \in \mathcal{L}\left(\left[L^{1}, B V_{0}\right]_{\theta},\left[L^{\infty}, \widehat{H K}\right]_{\theta}\right) .
$$

The following estimate for its norm is valid:

$$
\left\|\mathfrak{F}_{1}^{c}\right\|_{\mathcal{L}\left(\left[L^{1}, B V_{0}\right]_{\theta},\left[L^{\infty}, H K\right]_{\theta}\right)} \leq\left\|\mathcal{F}_{1}^{c}\right\|_{\mathcal{L}\left(L^{1}, L^{\infty}\right)}^{1-\theta}\left\|\mathcal{F}_{H K}^{c}\right\|_{\mathcal{L}\left(B V_{0}, H K\right)}^{\theta} \leq \mathfrak{C}^{\theta}
$$

for every $\theta \in(0,1)$, where

$$
\mathfrak{C}=4 \pi \operatorname{Si}(\pi) \quad \text { and } \quad \operatorname{Si}(x):=\frac{2}{\pi} \int_{0}^{x} \frac{\sin (y)}{y} d y .
$$

Furthermore, from Remark 3.5 we have that

$$
\left(L^{1}, B V_{0}\right)_{\theta, 1} \subset\left[L^{1}, B V_{0}\right]_{\theta} \subset\left(L^{1}, B V_{0}\right)_{\theta, \infty}
$$

holds for each $\theta \in(0,1)$.

Proposition 3.9. For $f \in\left[L^{1}, B V_{0}\right]_{\theta}$, the formula

$$
\mathfrak{F}_{1}^{c}(f)(s)=\int_{-\infty}^{\infty} \cos (s x) f(x) d x
$$

holds true pointwise almost everywhere and the Riemann-Lebesgue lemma is satisfied: $\mathfrak{F}_{1}^{c}(f)(s) \rightarrow 0$ as $|s| \rightarrow \infty$.

Proof. If $f=f_{1}+f_{0}=\tilde{f}_{1}+\tilde{f}_{0}$ belongs to $L^{1}(\mathbb{R}, \mathbb{C})+B V_{0}(\mathbb{R}, \mathbb{C})$, then

$$
\left(f_{1}-\tilde{f}_{1}, f_{0}-\tilde{f}_{0}\right) \in D^{\prime} .
$$

This yields $f_{1}-\tilde{f}_{1}=\tilde{f}_{0}-f_{0}$ with $f_{1}-\tilde{f}_{1} \in L^{1}(\mathbb{R}, \mathbb{C})$ and $\tilde{f}_{0}-f_{0} \in B V_{0}(\mathbb{R}, \mathbb{C})$.

Since $\mathcal{F}_{1}^{c}$ and $\mathcal{F}_{H K}^{c}$ coincide on $L^{1}(\mathbb{R}, \mathbb{C}) \cap B V_{0}(\mathbb{R}, \mathbb{C})$ due to 2.1 , we conclude that

$$
\mathcal{F}_{1}^{c}\left(f_{1}\right)+\mathcal{F}_{H K}^{c}\left(f_{0}\right)=\mathcal{F}_{1}^{c}\left(\tilde{f}_{1}\right)+\mathcal{F}_{H K}^{c}\left(\tilde{f}_{0}\right) .
$$

As a consequence, the value of $\mathfrak{F}_{1}^{c}(f)(s)$ does not depend on the representation of $f \in\left[L^{1}, B V_{0}\right]_{\theta}$, for each $\theta \in(0,1)$. From Theorem 3.6. for every $f \in\left[L^{1}, B V_{0}\right]_{\theta}$, there exist $f_{1} \in L^{1}(\mathbb{R}, \mathbb{C})$ and $f_{0} \in B V_{0}(\mathbb{R}, \mathbb{C})$ such that $f=f_{1}+f_{0}$ and for each 
$s \neq 0$,

$$
\begin{aligned}
\mathfrak{F}_{1}^{c}(f)(s) & =\mathfrak{F}^{c}\left(f_{1}+f_{0}\right)(s) \\
& =\mathcal{F}_{1}^{c}\left(f_{1}\right)(s)+\mathcal{F}_{H K}^{c}\left(f_{0}\right)(s) \\
& =\int_{-\infty}^{\infty} \cos (s x) f_{1}(x) d x+\int_{-\infty}^{\infty} \cos (s x) f_{0}(x) d x \\
& =\int_{-\infty}^{\infty} \cos (s x)\left(f_{1}(x)+f_{0}(x)\right) d x \\
& =\int_{-\infty}^{\infty} \cos (s x) f(x) d x .
\end{aligned}
$$

Then, (3.4) establishes that the HK-Fourier cosine transform on $\left[L^{1}, B V_{0}\right]_{\theta}$ has an integral representation. From the Riemann-Lebesgue lemma [21, Lemma 2] we conclude that $\mathfrak{F}_{1}^{c}(f)(s) \rightarrow 0$ as $|s| \rightarrow \infty$.

In [1, 8, the Sobolev spaces $W^{1, p}(\mathbb{R})$ are defined and for their complexification $W^{1, p}(\mathbb{R}, \mathbb{C}):=W^{1, p}(\mathbb{R})+i W^{1, p}(\mathbb{R})$ we have the next statement.

Lemma 3.10. For each $\theta \in(0,1)$,

$$
W^{1,1}(\mathbb{R}, \mathbb{C}) \subset\left[L^{1}(\mathbb{R}, \mathbb{C}), B V_{0}(\mathbb{R}, \mathbb{C})\right]_{\theta}
$$

with continuous inclusion.

Proof. First we recall that $W^{1,1}(\mathbb{R}) \subset \mathcal{L}^{1}(\mathbb{R}) \cap B V_{0}(\mathbb{R})$. From [4, Theorem 7.5] we have

$$
\begin{aligned}
\|u\|_{L^{1} \cap B V_{0}} & :=\max \left\{\|u\|_{L^{1}},\|u\|_{B V}\right\} \\
& \leq\|u\|_{L^{1}}+\|u\|_{B V} \\
& =\|u\|_{L^{1}}+\left\|u^{\prime}\right\|_{L^{1}} \\
& =\|u\|_{W^{1,1}} .
\end{aligned}
$$

If $u$ belongs to $W^{1,1}(\mathbb{R}, \mathbb{C})$, from [18, Proposition 2.4] we get

$$
\|u\|_{[\theta]} \leq \max \left\{\|u\|_{L^{1}},\|u\|_{B V}\right\} \leq\|u\|_{W^{1,1}(\mathbb{R}, \mathbb{C})},
$$

for every $\theta \in(0,1)$.

Corollary 3.11. For $u \in W^{1,1}(\mathbb{R}, \mathbb{C}), \mathfrak{F}_{1}^{c}(u)$ belongs to $H K(\mathbb{R}, \mathbb{C})$.

The proof of Corollary 3.11 follows from the fact that $W^{1,1}(\mathbb{R}) \subset B V_{0}(\mathbb{R})$, and then by Theorem 2.1 .

$$
\mathcal{F}_{H K}^{c}\left(W^{1,1}(\mathbb{R})\right) \subset H K(\mathbb{R}) .
$$

Therefore, the range of the Sobolev space $W^{1,1}(\mathbb{R}, \mathbb{C})$ under the HK-Fourier cosine transform is contained in $H K(\mathbb{R}, \mathbb{C})$. Explicitly,

$$
\mathfrak{F}_{1}^{c}\left(W^{1,1}(\mathbb{R}, \mathbb{C})\right) \subset H K(\mathbb{R}, \mathbb{C}) .
$$

The Fourier cosine and sine transforms are continuous operators on $L^{2}(\mathbb{R}, \mathbb{C})$, while their qualitative differences appear even on the space of functions $W^{1,1}(\mathbb{R}, \mathbb{C})$ that have a degree of regularity. In the following example we show this difference. 
Example 3.12. Let us define

$$
h(x):= \begin{cases}\frac{1}{2-\log (x)} & \text { if } x \in(0,1] \\ 0 & \text { if } x>1\end{cases}
$$

For each $x \in(0,1)$, we have

$$
h^{\prime}(x)=\frac{1}{x[2-\log (x)]^{2}} .
$$

We extend $h$ over $\mathbb{R}$ as an odd map. Also, we consider an even function $\varphi \in C_{c}^{\infty}(\mathbb{R})$ such that $0 \leq \varphi(x) \leq 1$, with $\varphi(x)=1$ for $|x| \leq 1 / 2$ and vanishing for $|x| \geq 1$. We define $f(x):=h(x) \varphi(x)$, for all $x \in \mathbb{R}$. Thus, $f$ is an odd map belonging to $W^{1,1}(\mathbb{R}) \subset L^{1}(\mathbb{R}) \cap B V_{0}(\mathbb{R})$ and

$$
\mathcal{F}_{H K}^{s}(f)(s)=2 \int_{0}^{\infty} \sin (s x) f(x) d x, \quad \text { for all } s \geq 0 .
$$

We analyze the convergence of the Henstock-Kurzweil integral:

$$
\int_{0}^{\infty} \mathcal{F}_{H K}^{s}(f)(s) d s .
$$

For any fixed $s>0$, the map $x \mapsto \sin (s x)$ belongs to $H K[0, M]$ for $0<M<\infty$, and we have

$$
\|\sin (s \cdot)\|_{H K[0, M]}=\sup _{0<u<v<M}\left|\int_{u}^{v} \sin (s t) d t\right| \leq \frac{2}{s} .
$$

Thus, for $0<b<\infty$, we get from Lebesgue's dominated convergence theorem, Fubini's theorem and Hake's theorem [4]:

$$
\int_{0}^{b} \int_{0}^{\infty} \sin (s x) f(x) d x d s=\lim _{M \rightarrow \infty} \int_{0}^{M} \frac{1-\cos (b x)}{x} f(x) d x .
$$

In fact,

$$
\int_{0}^{M} \frac{1-\cos (b x)}{x} f(x) d x=\int_{0}^{b} \frac{1-\cos (y)}{y} f(y / b) d y .
$$

Now, for $\delta=1 / 4$,

$$
\int_{0}^{b} \frac{1-\cos (y)}{y} f(y / b) d y=\int_{0}^{\delta} \frac{1-\cos (y)}{y} f(y / b) d y+\int_{\delta}^{b} \frac{1-\cos (y)}{y} f(y / b) d y .
$$

Since $f(y / b) \rightarrow 0$ as $b \rightarrow \infty$, we have that

$$
\lim _{b \rightarrow \infty} \int_{0}^{\delta} \frac{1-\cos (y)}{y} f(y / b) d y=0 .
$$

For the second integral on the right side of (3.7) we have

$$
\int_{\delta}^{b} \frac{1-\cos (y)}{y} f(y / b) d y=\int_{\delta}^{b} \frac{f(y / b)}{y} d y+\int_{\delta}^{b} \frac{-\cos (y)}{y} f(y / b) d y=I_{1}+I_{2} .
$$


Integrating by parts,

$$
\begin{aligned}
\lim _{b \rightarrow \infty} I_{2} & =\lim _{b \rightarrow \infty}-\int_{\delta}^{b}-\sin (y)\left(\frac{b^{-1} y f^{\prime}(y / b)-f(y / b)}{y^{2}}\right) d y \\
& =\lim _{b \rightarrow \infty} \int_{\delta / b}^{1} \frac{\sin (b t)}{b t} f^{\prime}(t) d t-\int_{\delta}^{b} \frac{\sin (y) f(y / b)}{y^{2}} d y .
\end{aligned}
$$

By Lebesgue's dominated convergence theorem we conclude that

$$
\lim _{b \rightarrow \infty} \int_{\delta}^{b} \frac{\sin (y) f^{\prime}(y / b)}{b y} d y=\lim _{b \rightarrow \infty} \int_{\delta}^{b}-\frac{\sin (y)}{y^{2}} f(y / b) d y=0 .
$$

Therefore the limit of $I_{2}$ is zero. Writing explicitly the integrand of the integral $I_{1}$ we get

$$
I_{1}=\int_{\delta / b}^{1 / 2} \frac{1}{u[2-\log (u)]}+\int_{1 / 2}^{1} \frac{\varphi(u)}{u[2-\log (u)]} d u
$$

and

$$
\lim _{b \rightarrow \infty} \int_{\delta / b}^{1 / 2} \frac{1}{u[2-\log (u)]} d u=\infty .
$$

Therefore, the integral in 3.6 does not exist and $\mathcal{F}_{H K}^{s}(f)$ does not belong to $H K(\mathbb{R})$. In conclusion,

$$
\mathcal{F}_{2}^{s}\left(W^{1,1}\right)=\mathcal{F}_{1}^{s}\left(W^{1,1}\right)=\mathcal{F}_{H K}^{s}\left(W^{1,1}(\mathbb{R})\right) \nsubseteq H K(\mathbb{R})
$$

Then, the HK-Fourier sine transform remains unbounded on $W^{1,1}(\mathbb{R})$, in contrast with relation (3.5). Also, in [3, Example 1] it was established that

$$
\mathcal{F}_{H K}^{s}\left(B V_{0}(\mathbb{R}) \backslash W^{1,1}(\mathbb{R})\right) \nsubseteq H K(\mathbb{R}) \text {. }
$$

The function given in Example 3.12 is a slight variation of one considered in [11.

We can proceed in the same way to define the space $L^{2}(\mathbb{R}, \mathbb{C})+B V_{0}(\mathbb{R}, \mathbb{C})$. Therefore, we have the continuous inclusions

$$
L^{2}(\mathbb{R}, \mathbb{C}) \cap B V_{0}(\mathbb{R}, \mathbb{C}) \subset\left[L^{2}(\mathbb{R}, \mathbb{C}), B V_{0}(\mathbb{R}, \mathbb{C})\right]_{\theta} \subset L^{2}(\mathbb{R}, \mathbb{C})+B V_{0}(\mathbb{R}, \mathbb{C}),
$$

for $0<\theta<1$. Now for the extended operators $\mathcal{F}_{2}^{c}$ and $\mathcal{F}_{H K}^{c}$ on $L^{2}(\mathbb{R}, \mathbb{C})$ and on $B V_{0}(\mathbb{R}, \mathbb{C})$ respectively, we define the map

$$
\begin{aligned}
& \mathfrak{F}_{2}^{c}: L^{2}(\mathbb{R}, \mathbb{C})+B V_{0}(\mathbb{R}, \mathbb{C}) \longrightarrow L^{2}(\mathbb{R}, \mathbb{C})+\widehat{H K}(\mathbb{R}, \mathbb{C}) \\
& \mathfrak{F}_{2}^{c}(f+g):=\mathcal{F}_{2}^{c}(f)+\mathcal{F}_{H K}^{c}(g) .
\end{aligned}
$$

So, by Theorem 3.6, we have

$$
\mathfrak{F}_{2}^{c} \in \mathcal{L}\left(\left[L^{2}, B V_{0}\right]_{\theta},\left[L^{2}, \widehat{H K}\right]_{\theta}\right),
$$

with the following estimate for its norm:

$$
\left\|\mathfrak{F}_{2}^{c}\right\|_{\mathcal{L}\left(\left[L^{2}, B V_{0}\right]_{\theta},\left[L^{2}, \widehat{H K}\right]_{\theta}\right)} \leq\left\|\mathcal{F}_{2}^{c}\right\|_{\mathcal{L}\left(L^{2}, L^{2}\right)}^{1-\theta}\left\|\mathcal{F}_{H K}^{c}\right\|_{\mathcal{L}\left(B V_{0}, \widehat{H K}\right)}^{\theta} \leq(2 \pi)^{\frac{1-\theta}{2}} \mathfrak{C}^{\theta}
$$


for every $\theta \in(0,1)$ and $\mathfrak{C}$ given by $(3.3)$.

Similarly, for the operators $\mathcal{F}_{p}^{c}$ and $\mathcal{F}_{H K}^{c}$ on $L^{p}(\mathbb{R}, \mathbb{C})$ and on $B V_{0}(\mathbb{R}, \mathbb{C})$ respectively, we define

$$
\begin{aligned}
& \mathfrak{F}_{p}^{c}: L^{p}(\mathbb{R}, \mathbb{C})+B V_{0}(\mathbb{R}, \mathbb{C}) \longrightarrow L^{q}(\mathbb{R}, \mathbb{C})+\widehat{H K}(\mathbb{R}, \mathbb{C}) \\
& \mathfrak{F}_{p}^{c}(f):=\mathcal{F}_{p}^{c}\left(f_{p}\right)+\mathcal{F}_{H K}^{c}(g),
\end{aligned}
$$

where $f=f_{p}+g$ with $1 / p+1 / q=1$. This operator is a generalization of the map considered in [3, Corollary 1]. For the couples given by $X_{1}=L^{p}(\mathbb{R}, \mathbb{C})$ and $X_{2}=L^{q}(\mathbb{R}, \mathbb{C})$, with $1 \leq p \leq 2$, and $Y_{1}=B V_{0}(\mathbb{R}, \mathbb{C})$ and $Y_{2}=\widehat{H K}(\mathbb{R}, \mathbb{C})$ we have from Theorem 3.6 that

$$
\mathfrak{F}_{p}^{c} \in \mathcal{L}\left(\left[L^{p}, B V_{0}\right]_{\theta},\left[L^{q}, \widehat{H K}\right]_{\theta}\right)
$$

for every $\theta \in(0,1)$, and the following estimate for the norm:

$$
\left\|\mathfrak{F}_{p}^{c}\right\|_{\mathcal{L}\left(\left[L^{p}, B V_{0}\right]_{\theta},\left[L^{q}, \widehat{H K}\right]_{\theta}\right)} \leq\left\|\mathcal{F}_{p}^{c}\right\|_{\mathcal{L}\left(L^{p}, L^{q}\right)}^{1-\theta}\left\|\mathcal{F}_{H K}^{c}\right\|_{\mathcal{L}\left(B V_{0}, \widehat{H K}\right)}^{\theta} \leq \gamma_{p}^{1-\theta} \mathfrak{C}^{\theta}
$$

where $\mathfrak{C}$ is given in 3.3 .

For $1<p<2$, the relation between $\mathfrak{F}_{p}^{c}, \mathfrak{F}_{1}^{c}$ and $\mathfrak{F}_{2}^{c}$ is given by the decomposition of $L^{p}(\mathbb{R})$ in $\left[23\right.$, which implies that for each $f_{p}+g \in L^{p}(\mathbb{R})+B V_{0}(\mathbb{R})$ there exist $f_{1} \in L^{1}(\mathbb{R}) \cap L^{p}(\mathbb{R})$ and $f_{2} \in L^{2}(\mathbb{R}) \cap L^{p}(\mathbb{R})$ such that

$$
f_{p}+g=\left(f_{1}+g\right)+f_{2}=f_{1}+\left(f_{2}+g\right) .
$$

Corollary 3.13. For $1<p<2$, $\mathfrak{F}_{p}^{c}\left(f_{p}+g\right)=\mathfrak{F}_{1}^{c}\left(f_{1}+g\right)+\mathfrak{F}_{2}^{c}\left(f_{2}\right)$.

Proof. This follows by taking a sequence $\left(f_{n}\right)_{n \geq 1}$ on $L^{p}(\mathbb{R})$ such that $f_{n} \rightarrow f$ with $f=f_{1}+f_{2} \in L^{p}(\mathbb{R})$, and using that the sequence $f_{n}=f_{1, n}+f_{2, n}$ has the property $f_{i, n} \rightarrow f_{i}$ in the norm of $L^{i}(\mathbb{R}), i=1,2$; see $[23$.

Proposition 3.14. For $f \in W^{1, p}(\mathbb{R})$ with $1<p \leq 2$, the formula

$$
\mathcal{F}_{p}\left(f^{\prime}\right)(s)=i s \mathcal{F}_{p}(f)(s)
$$

holds true pointwise almost everywhere.

Proof. If $f \in W^{1, p}(\mathbb{R})$ then $f, f^{\prime}$ belong to $L^{p}(\mathbb{R})$ with $f(x) \rightarrow 0$ as $|x| \rightarrow \infty$; see [8, Corollary 8.9]. Next, for each $n \geq 1$, we let $\varphi_{n}(x):=\chi_{[-n, n]}(x) f(x)$ and $\gamma_{n}(x):=\chi_{[-n, n]} f^{\prime}(x)$. So $\left\|\varphi_{n}-f\right\|_{L^{p}} \rightarrow 0$ as $n \rightarrow \infty$, and there exists a subsequence $\left(\varphi_{n_{k}}\right)_{k \geq 1}$ such that $\mathcal{F}_{p}\left(\varphi_{n_{k}}\right)(s) \rightarrow \mathcal{F}_{p}(f)(s)$ a.e. as $k \rightarrow \infty$. Therefore,

$$
\mathcal{F}_{p}(f)(s)=\lim _{k \rightarrow \infty} \mathcal{F}_{p}\left(\varphi_{n_{k}}\right)(s)=\lim _{k \rightarrow \infty} \int_{-n_{k}}^{n_{k}} e^{-i s x} f(x) d x
$$

almost everywhere. Integrating by parts [8, Corollary 8.10], for each $k \geq 1$,

$$
\int_{-n_{k}}^{n_{k}} e^{-i s x} f^{\prime}(x) d x=\left.e^{-i s x} f(x)\right|_{-n_{k}} ^{n_{k}}-\int_{-n_{k}}^{n_{k}}-i s e^{-i s x} f(x) d x .
$$


Thus,

$$
\begin{aligned}
\mathcal{F}_{p}\left(f^{\prime}\right)(s) & =\lim _{k \rightarrow \infty} \mathcal{F}_{p}\left(\gamma_{n_{k}}\right)(s)=\lim _{k \rightarrow \infty} \int_{-n_{k}}^{n_{k}} e^{-i s x} f^{\prime}(x) d x \\
& =i s \lim _{k \rightarrow \infty} \int_{-n_{k}}^{n_{k}} e^{-i s x} f(x) d x=i s \mathcal{F}_{p}(f)(s)
\end{aligned}
$$

almost everywhere.

Proposition 3.15. If $f \in W^{1, p}(\mathbb{R})$ with $1<p \leq 2$, then $\mathcal{F}_{p}(f) \in L^{1}(\mathbb{R})$.

Proof. If $f \in W^{1, p}(\mathbb{R})$ then $\mathcal{F}_{p}(f)$ belongs to $L^{q}(\mathbb{R})$ with $1 / p+1 / q=1$ and for $A=\{s \in \mathbb{R}:|s| \geq 1\}$ we get, by Proposition 3.14 and Hölder's inequality,

$$
\int_{A}\left|\mathcal{F}_{p}(f)(s)\right| d s=\int_{A}\left|\frac{1}{s} \mathcal{F}_{p}\left(f^{\prime}\right)(s) d s\right| \leq\|1 /(\cdot)\|_{L^{p}(A)}\left\|\mathcal{F}_{p}\left(f^{\prime}\right)\right\|_{L^{q}(A)}<\infty,
$$

with $1 / p+1 / q=1$. Therefore, $\mathcal{F}_{p}(f) \in L^{1}(\mathbb{R})$.

As a consequence of Proposition 3.15, the range of $W^{1, p}(\mathbb{R})$, for $1<p \leq 2$, under the action of the $L^{p}$-Fourier transform operator is contained in $L^{1}(\mathbb{R})$. Explicitly,

$$
\mathcal{F}_{p}\left(W^{1, p}(\mathbb{R})\right) \subset L^{1}(\mathbb{R}) \subsetneq H K(\mathbb{R}) .
$$

This relation contrasts with 3.8 .

\section{REFERENCES}

[1] R. A. Adams and J. J. F. Fournier, Sobolev Spaces, second edition, Elsevier/Academic Press, Amsterdam, 2003. MR 2424078

[2] A. Alexiewicz and W. Orlicz, Analytic operations in real Banach spaces, Studia Math. 14 (1953), 57-78. MR 0062947.

[3] J. H. Arredondo, F. J. Mendoza and A. Reyes, On the norm continuity of the HK-Fourier transform, Electron. Res. Announc. Math. Sci. 25 (2018), 36-47. MR 3810181

[4] R. G. Bartle, A Modern Theory of Integration, Graduate Studies in Mathematics, 32, American Mathematical Society, Providence, RI, 2001. MR 1817647

[5] W. Beckner, Inequalities in Fourier analysis on $R^{n}$, Proc. Nat. Acad. Sci. U.S.A. 72 (1975), 638-641. MR 0385455

[6] J. Bergh and J. Löfström, Interpolation Spaces. An Introduction, Grundlehren der Mathematischen Wissenschaften, No. 223, Springer-Verlag, Berlin, 1976. MR 0482275

[7] B. Bongiorno and T. V. Panchapagesan, On the Alexiewicz topology of the Denjoy space, Real Anal. Exchange 21 (1995/96), no. 2, 604-614. MR 1407272

[8] H. Brezis, Functional Analysis, Sobolev Spaces and Partial Differential Equations, Universitext, Springer, New York, 2011. MR 2759829

[9] Yu. A. Brudnyı̌ and N. Ya. Krugljak, Interpolation Functors and Interpolation Spaces. Vol. I, translated from the Russian by Natalie Wadhwa, North-Holland Mathematical Library, 47, North-Holland, Amsterdam, 1991. MR 1107298

[10] A. P. Calderón, Intermediate spaces and interpolation, the complex method, Studia Math. 24 (1964), 113-190. MR 0167830

[11] Construct a function $F: X \rightarrow \mathbb{R}$ such that $F \in L_{p}(\mu)$ for all $p>1$, but $F \notin L_{1}(\mu)$, Mathematics Stack Exchange, 2014. https://math.stackexchange.com/q/843665

[12] R. A. Gordon, The Integrals of Lebesgue, Denjoy, Perron, and Henstock, Graduate Studies in Mathematics, 4, American Mathematical Society, Providence, RI, 1994. MR 1288751 
[13] S. G. Krĕ̌n, Yu. Petunīn and E. M. Semënov, Interpolation of Linear Operators, translated from the Russian by J. Szücs, Translations of Mathematical Monographs, 54, American Mathematical Society, Providence, RI, 1982. MR 0649411

[14] P. Y. Lee, Lanzhou Lectures on Henstock Integration, Series in Real Analysis, 2, World Scientific, Teaneck, NJ, 1989. MR 1050957

[15] E. H. Lieb and M. Loss, Analysis, Graduate Studies in Mathematics, 14, American Mathematical Society, Providence, RI, 1997. MR 1415616

[16] E. Liflyand, Integrability spaces for the Fourier transform of a function of bounded variation, J. Math. Anal. Appl. 436 (2016), no. 2, 1082-1101. MR 3446998

[17] M. E. Luna-Elizarrarás, F. Ramírez-Reyes and M. Shapiro, Complexifications of real spaces: general aspects, Georgian Math. J. 19 (2012), no. 2, 259-282. MR 2929656.

[18] A. Lunardi, Interpolation Theory, third edition, Appunti. Scuola Normale Superiore di Pisa (Nuova Serie), 16, Edizioni della Normale, Pisa, 2018. MR 3753604

[19] R. M. McLeod, The Generalized Riemann Integral, Carus Mathematical Monographs, 20, Mathematical Association of America, Washington, DC, 1980. MR 0588510

[20] F. J. Mendoza Torres, On pointwise inversion of the Fourier transform of $\mathrm{BV}_{0}$ functions, Ann. Funct. Anal. 1 (2010), no. 2, 112-120. MR 2772044

[21] F. J. Mendoza Torres and M. G. Morales Macías, On the convolution theorem for the Fourier transform of $B V_{0}$ functions, J. Class. Anal. 7 (2015), no. 1, 63-71. MR 3450960

[22] F. J. Mendoza Torres et al., Several aspects around the Riemann-Lebesgue lemma, J. Adv. Res. Pure Math. 5 (2013), no. 3, 33-46. MR 3041342

[23] M. G. Morales, J. H. Arredondo and F. J. Mendoza, An extension of some properties for the Fourier transform operator on $L^{p}(\mathbb{R})$ spaces, Rev. Un. Mat. Argentina 57 (2016), no. 2, 85-94. MR 3583297.

[24] G. A. Muñoz-Fernández, Y. Sarantopoulos and A. Tonge, Complexifications of real Banach spaces, polynomials and multilinear maps, Studia Math. 134 (1999), no. 1, 1-33. MR 1688213

[25] M. Reed and B. Simon, Methods of Modern Mathematical Physics. II, Academic Press, New York, 1975. MR 0493420

[26] M. Reed and B. Simon, Methods of Modern Mathematical Physics. I, second edition, Academic Press, New York, 1980. MR 0751959

[27] W. Rudin, Real and Complex Analysis, third edition, McGraw-Hill, New York, 1987. MR 0924157

[28] M. Schechter, Complex interpolation, Compositio Math. 18 (1967), 117-147. MR 0223880.

[29] P. Sjölin, A remark on the Hausdorff-Young inequality, Proc. Amer. Math. Soc. 123 (1995), no. 10, 3085-3088. MR 1273525

[30] E. Talvila, Henstock-Kurzweil Fourier transforms, Illinois J. Math. 46 (2002), no. 4, 12071226. MR 1988259

[31] E. Talvila, Convolutions with the continuous primitive integral, Abstr. Appl. Anal. 2009, Art. ID 307404, 18 pp. MR 2559282

\section{J. H. Arredondo}

Departamento de Matemáticas, Universidad Autónoma Metropolitana - Iztapalapa

Av. San Rafael Atlixco 186, 09340 Iztapalapa, CDMX, México

iva@xanum. uam.mx

A. Reyes ${ }^{凶}$

Departamento de Matemáticas, Universidad Autónoma Metropolitana - Iztapalapa

Av. San Rafael Atlixco 186, 09340 Iztapalapa, CDMX, México

alfredreuam@xanum.uam.mx

Received: February 3, 2020

Accepted: July 9, 2020 